\title{
Evidence for unique threat-processing mechanisms in psychopathic and anxious individuals
}

\author{
Allison J. Lake - Arielle R. Baskin-Sommers • Wen Li • \\ John J. Curtin - Joseph P. Newman
}

Published online: 18 May 2011

(C) Psychonomic Society, Inc. 2011

\begin{abstract}
Behaviorally, psychopathy and anxiety display opposite patterns of threat sensitivity and response inhibition. However, it is unclear whether this is due to shared or to separate underlying processes. To address this question, we evaluated whether the threat sensitivity of psychopathic and anxious offenders relates to similar or different components of Gray and McNaughton's (2000) Reinforcement Sensitivity Theory using a sample of 87 prisoners and a task that crossed threat onset with attentional focus. Psychopathy was associated with significantly weaker fearpotentiated startle (FPS) under conditions that presented threat cues after alternative, goal-directed cues. Conversely, anxiety was associated with significantly stronger FPS when threat appeared first and was the focus of attention. Furthermore, these differences were statistically independent. The results suggest that the abnormal sensitivity to threat cues associated with psychopathy and anxiety relate to different underlying processes and have implications for understanding the relationship between low- and highanxious psychopathy.
\end{abstract}

Keywords Anxiety· Psychopathy Behavioral inhibition

Historically, psychopathy and anxiety have been regarded as opposite constructs. In his seminal description of psychopathy, Cleckley (1976) described the "true" psychopath as lacking nervous thoughts and being "incapable of anxiety" (p. 340). In contrast with the overly inhibited

A. J. Lake $\cdot$ A. R. Baskin-Sommers $(\triangle) \cdot$ W. Li $\cdot$ J. J. Curtin • J. P. Newman

Department of Psychology, University of Wisconsin-Madison, 1202 W. Johnson St,

Madison, WI 53706, USA

e-mail: baskinsommer@wisc.edu behavioral style and hypersensitivity to threat stimuli that characterizes anxious individuals (e.g. MacLeod, Mathews, \& Tata, 1986; Mogg \& Bradley, 1998; Williams, Mathews, \& MacLeod, 1996), psychopathic individuals appear to be uninhibited and unconcerned with threat stimuli or the negative consequences of their actions (i.e., Fowles, 1980; Hare, 1965). Although psychopathy and anxiety appear to anchor opposite ends of a continuum involving threat sensitivity and response inhibition, research has yet to establish the true nature of this relationship. More specifically, it is unclear whether the opposite behavioral styles of psychopathic and anxious individuals reflect different psychobiological mechanisms or opposite extremes of the same psychobiological mechanism.

Notably, Fowles $(1980,2001)$ has suggested that psychopathy and anxiety involve a common mechanism operating in different directions. More specifically, he proposed that psychopathy as well as anxiety are related to Gray's $(1975,1982)$ neuropsychological behavioral inhibition system (BIS). Whereas Gray associated anxiety with excessive BIS activity, Fowles proposed that psychopathy was associated with deficient BIS activity. To date, support for the weak BIS model of psychopathy has been equivocal (e.g., Arnett, Smith, \& Newman, 1997; Baskin-Sommers, Wallace, MacCoon, Curtin, \& Newman, 2010; Newman, Wallace, Schmitt, \& Arnett, 1997; Ross, Molto, Poy, Segarra, Pastor \& Montanes, 2007; Schmitt \& Newman, 1999). However, the majority of this research was based on the premise, outlined in Gray's early work (e.g., Gray, 1982), that the BIS was the primary source of individual differences in threat sensitivity.

Gray and McNaughton (2000) published a revised Reinforcement Sensitivity Theory (RST; see also Corr, 2008) that reassigned the threat-sensitivity component of the BIS to a fight-flight-freezing system (FFFS). In the revised model, the FFFS is activated by novel stimuli, cues 
for punishment and nonreward, and motivates escape or avoidance behaviors. Accordingly, the role of the BIS was modified. Specifically, in the revised model, the BIS does not play a major role in threat processing unless the threat stimuli are encountered in the context of approach behavior (i.e., in situations involving approach-avoidance conflicts). Once activated, the conflict detector component of the BIS registers the conflict and inhibits both the avoidance and approach behaviors in order to process and resolve the conflict. According to Gray and McNaughton, the BIS itself does not control processing of threat stimuli. Rather, it functions like an attentional gating mechanism to increase the saliency and amount of attention devoted to threatening or conflicting cues when they occur in the context of approach behavior. ${ }^{1}$

Paralleling the role of attention in BIS processing during approach-avoidance conflicts (Gray \& McNaughton, 2000), the response modulation theory of psychopathy (Newman \& Lorenz, 2003; Patterson \& Newman, 1993) emphasizes the reallocation of attention in the context of goal-related behavior. According to this model, psychopathic individuals are not inherently impervious to threat cues, but they are less likely to switch their focus of attention to process affective and inhibitory cues that are peripheral to their goal-directed behavior. This characterization of psychopathy is consistent with Gray and McNaughton's model of weak BIS functioning. Relative to controls, psychopathic offenders are less likely to inhibit behavior or increase attention to threat-related stimuli in approach-avoidance situations (Wallace \& Newman, 2008).

To investigate the importance of attentional processes in moderating the fearlessness of psychopathic offenders, Newman, Curtin, Bertsch, and Baskin-Sommers (2010) examined fear potentiated startle (FPS) under three conditions that manipulated the relevancy of threat to goaldirected behavior. In the threat-focus (TF) condition, participants categorized colored letter stimuli according to the threat-relevant aspect of the stimuli (i.e., letter color predicted administration of electric shock). In two alternative focus (AF) conditions, participants responded accord-

\footnotetext{
${ }^{1}$ In this context, it should be noted that Gray $(1972,1987)$ referred to his proposals as a conceptual nervous system (i.e., cNS) to highlight the fact that they represented only a set of tentative theoretical hypotheses to guide research on actual central nervous system function. For the most part, these hypotheses were based on drug and brain lesion studies conducted with rats rather than human beings. Following Gray, and consistent with his tentative hypothesis generating perspective on the cNS, we assume that current understanding of the BIS, FFFS, and other hypothetical systems will require revisions as the quality of existing evidence improves. In mapping psychopathy and anxiety onto different components of the RST model, it is not our intention to reify the specific mappings within the RST model. Rather, we use the RST model as a heuristic to conceptualize the core processes that underlie the threat sensitivity differences apparent in psychopathy and anxiety.
}

ing to an alternative, threat-irrelevant aspect of the stimuli (i.e., the threat-relevant information was outside the primary focus of goal-directed attention). In the alternative-focus/low load (AF/LL) condition, participants indicated whether letters were upper- or lower case. In the alternative-focus/high load (AF/HL) condition, participants had to monitor the sequence of letters and indicate whether each letter matched or mismatched the letter that had appeared two letters back (i.e., two-back working memory task). Because psychopathy-related differences were comparable in the two AF conditions, the data from these conditions were combined to yield a $\mathrm{TF}$ versus $\mathrm{AF}$ comparison.

As was predicted, psychopathy was significantly associated with reduced FPS when threat information was outside the goal-directed set (i.e. the two AF conditions), but it was unrelated to FPS under conditions that focused attention on the threat-relevant dimension (i.e. TF). These results suggest that psychopathy-related abnormalities in attention undermine sensitivity to emotion-related cues, specifically when they are peripheral to their goal-directed behavior. Moreover, the psychopathy-related abnormality was specific to the conditions in which threat cues were competing with approach cues (e.g., the identity of the letter). Under these conditions, participants with low levels of psychopathy interrupted their goal-directed focus (i.e., approach behavior) and reallocated attention to peripheral threat-related stimuli, suggesting normal BIS functioning. Conversely, those with high levels of psychopathy remained focused on approach and appeared oblivious to the peripheral threat cues. With regard to the RST framework, the findings for psychopathy are consistent with Gray and McNaughton's (2000) characterization of weak BIS functioning, which emphasizes the competition between threat processing and goal-directed behavior rather than threat sensitivity, per se.

Using the same paradigm, Dvorak-Bertsch, Curtin, Rubinstein, and Newman (2007) examined the association between trait anxiety and FPS in a sample of undergraduate students. Paralleling the findings for psychopathic individuals, high- and low-anxious participants displayed comparable FPS in the TF condition. However, in contrast with Newman et al.'s (2010) findings, the anxiety-related differences were specific to the AF/LL condition; there were no differences between groups in the AF/HL condition. Specifically, whereas psychopathy was associated with reduced FPS in the AF conditions, anxiety was associated with greater FPS in the $\mathrm{AF} / \mathrm{LL}$ condition. This suggests that although psychopathy is associated with reduced ability to suspend goal-directed behavior and to switch the focus of attention to peripheral threat information, anxiety is associated with a greater tendency to do so under similar circumstances (i.e., in the AF/LL condition). Thus, one interpretation of these findings is that psychopathy and anxiety may be conceptu- 
alized as anchoring opposite ends of a continuum that reflects individual differences in BIS functioning as defined by the Gray and McNaughton (2000) model.

Whereas the Dvorak-Bertsch et al. (2007) study found anxiety-related differences in the attention-switching component of BIS functioning, other research suggests that trait anxiety is associated with a pervasive perceptual bias that increases sensitivity to punishment-related stimuli. For example, Li, Zinbarg, Boehm, and Paller (2008) proposed that trait anxiety is associated with a preattentional, unconscious sensitivity for threat detection that subsequently pulls the focus of attention toward threat cues (see also Mathews \& MacLeod, 2002, 2005; Williams et al., 1996). In contrast with the findings of Dvorak-Bertsch et al., this group of findings indicates that trait anxiety is associated with a perceptual bias to focus on threat cues regardless of approach-related considerations. Within the RST framework, such findings suggest that the heightened threat sensitivity associated with trait anxiety "affects the FFFS directly, and the BIS (only) indirectly (e.g., via FFFS-BAS goal conflict)" (see Corr, 2008, p. 16). According to this view, individual differences in threat sensitivity associated with psychopathy and trait anxiety may be associated with different psychobiological processes (i.e., weak BIS and strong FFFS activation, respectively) (see Fig. 1).

In light of these alternative models and findings, there is a need for additional research to clarify the factors responsible for the threat-processing anomalies displayed by psychopathic and anxious individuals. Although Dvorak-Bertsch et al. (2007) suggested that anxious participants' heightened FPS in the $\mathrm{AF} / \mathrm{LL}$ condition reflected excessive reallocation of attention from task-relevant stimuli to process threat cues, their study could not differentiate such an attentional mechanism from an alternative explanation involving a pervasive perceptual threat bias of the type suggested by $\mathrm{Li}$ et al. (2008) and associated with the FFFS (Corr, 2008). Specifically, because threat and goal cues were integrated into the same stimuli (i.e. letter color and letter case), it was not possible to separate participants' reactions to the threat versus goal-relevant components of the stimuli in the $\mathrm{AF}$ conditions. Thus, further research is needed to evaluate the extent to which anxiety involves an attentional reallocation mechanism, such as psychopathy, or a perceptual bias involving heightened sensitivity to threat cues. In other words, it remains unclear whether the differential threat sensitivity associated with psychopathy and anxiety is better understood as diverse manifestations of a common mechanism or as a reflection of independent RST processes. To address this question, we use a task that enables us to evaluate threat sensitivity when (a) threat stimuli are the direct focus of attention or (b) threat information is peripheral to the primary focus of attention and attending to it requires participants to interrupt ongoing goal-directed behavior.
Baskin-Sommers, Curtin, and Newman (2011) recently developed such a task to clarify the nature of the fear deficits associated with psychopathy. Specifically, these authors examined FPS using a task that made attending to threat stimuli either primary or peripheral to goal-directed behavior. In addition, the authors manipulated the timing of the threat information so that threat stimuli were presented before or after threat-irrelevant stimuli. As was predicted, psychopathic individuals displayed a significant deficit in FPS when threat cues were presented after the goal-directed focus was established (i.e., early alternative-focus condition). Moreover, replicating earlier findings reported by Newman et al. (2010), psychopathic and nonpsychopathic individuals displayed comparable FPS when threat was their primary focus of attention. Thus, resembling the consequences of a weak BIS in the revised model, psychopathy was associated with a situation-specific failure to reallocate attention to process threat information, specifically when they are engaged in goal-directed behavior.

The overall purpose of the present study was to evaluate whether the differential sensitivity to threat stimuli associated with psychopathy and anxiety is better understood as opposite ends of a unitary continuum or as a reflection of fundamentally different processes. Toward this end, we reanalyzed the data reported by Baskin-Sommers et al. $(2011),{ }^{2}$ this time analyzing the effects of psychopathy and anxiety simultaneously, in order to determine whether their threat-related responses (i.e., FPS) relate to shared (i.e., overlapping) or independent (i.e., unique) variance. If psychopathy and anxiety are related to a common mechanism functioning in opposite directions, then they should be associated with the same variance in task performance, albeit in opposite directions. That is, anxious individuals should show greater FPS in the same condition that revealed psychopathy-related deficits in FPS. Furthermore, to the extent that the behavior of psychopathic and anxious individuals reflects a common mechanism, their effects will involve common variance, and the effect of anxiety would be expected to mediate the psychopathy effect reported by Baskin-Sommers et al. On the other hand, if psychopathy and anxiety reflect independent mechanisms in the revised RST framework, as has been suggested by recent anxiety findings, then their significant effects on FPS should account for unique variance in task performance and predict distinctive performance under different circumstances. That

\footnotetext{
${ }^{2}$ Although the data for psychopathy have been published in this previous article, we believe that the re-use of these data to evaluate the effects of trait anxiety and their relation to the psychopathy findings is justified by the importance of the additional questions. We did not combine these analyses in our original publication because the theoretical questions addressed in that article are distinct from those addressed in the present article.
} 


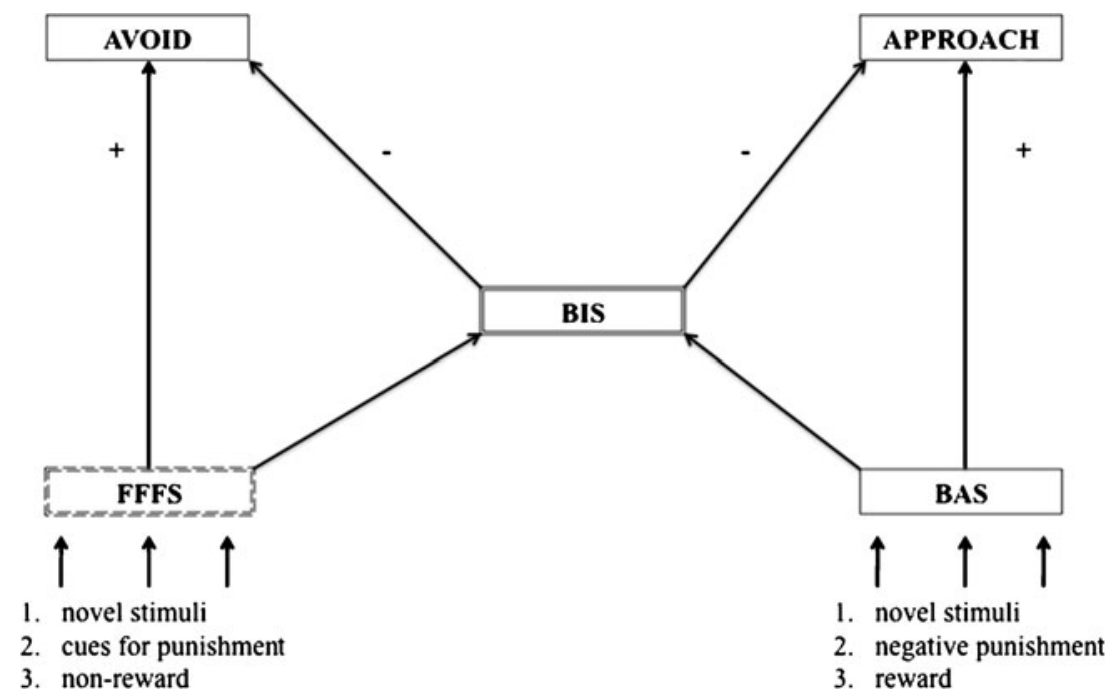

Fig. 1 Anxiety and psychopathy in relation to Gray and McNaughton (2000). Modified from Gray and McNaughton. The FFFS is activated by inputs of novel stimuli, cues for punishment, and nonreward, and motivates escape or avoidance behaviors. In contrast, the BAS is activated by inputs of novel stimuli, negative punishment, and cues for reward and motivates approach behavior. The BIS is activated when both the FFFS and BAS are activated, as in approach-avoidance conflicts. Once activated, the BIS registers the conflict and inhibits both the avoidance and approach signals from the FFFS and BAS, respectively, in order to process and resolve the conflict. As a result,

is, the effects of psychopathy on FPS will be most apparent after they are engaged in goal-directed behavior as reported by Baskin-Sommers et al. (i.e., resemble a weak BIS in the revised RST model), but the effects of anxiety will be most apparent when threat cues constitute participants' primary focus of attention (i.e., resemble a reactive FFFS in the revised RST model). Furthermore, if anxiety and psychopathy are independent, it is possible, though not necessarily the case, that anxiety will moderate the psychopathy effect (i.e., just as FFFS and BIS may interact to influence threat sensitivity under certain circumstances).

\section{Method}

\section{Participants}

Participants were the same sample of 87 EuropeanAmerican male inmates from the Baskin-Sommers et al. (2011) study. Participants whose institutional files indicated no record of schizophrenia, bipolar disorder, psychosis NOS diagnoses, or psychotropic medication use were recruited for this study (Cleckley, 1976; Hare, Harpur \& Hakstian, 1990; Hart \& Hare, 1989; Zachary, 1986). Furthermore, participants were required to be 45 years or younger and to score 70 or better on the Shipley estimate of intelligence. The Dodge Correctional Institution is a the BIS increases arousal and attention specifically toward negative cues that may resolve the approach-avoidance conflict. Thus, the BIS serves as an attentional gating mechanism that increases the saliency of threatening or conflicting cues and subsequently increases the amount of attentional resources devoted to such cues. According to our hypotheses, anxiety reflects a perceptual bias toward these inputs to the FFFS (double-dashed line). Alternatively, psychopathy reflects a difficulty in the reorientation of attention, which is a process of the BIS (double solid line)

maximum-security prison and the primary intake facility for the State of Wisconsin. Consequently, we had access to a complete cross-section of male offenders that included violent as well as nonviolent and first-time as well as repeat offenders. Descriptive statistics are reported in Table 1. Participants were paid at least $\$ 15$ for completing the diagnostic interview and a battery of self-report questionnaires. They received a minimum of $\$ 25$ for completing the full psychophysiological session.

Table 1 Descriptive statistics for relevant variables $(N=87)$

\begin{tabular}{lllll}
\hline Variable & Mean & $\begin{array}{l}\text { Standard } \\
\text { Deviation }\end{array}$ & Minimum & Maximum \\
\hline Age & 31.64 & 7.30 & 18.00 & 45.00 \\
PCL-R Total & 23.22 & 7.21 & 6.00 & 36.00 \\
PCL-R Factor 1 & 8.33 & 3.34 & 2.00 & 16.00 \\
PCL-R Factor 2 & 12.70 & 4.20 & 2.00 & 18.00 \\
Shipley & 102.03 & 10.31 & 71.00 & 118.91 \\
WAS & 14.56 & 10.04 & 0.00 & 37.00 \\
SR & 6.20 & 3.81 & 0.00 & 12.00 \\
Number of violent crimes & 3.56 & 3.34 & 0.00 & 15.00 \\
Number of nonviolent & 16.51 & 14.55 & 0.00 & 95.00 \\
$\quad$ crimes & & & & \\
\end{tabular}

PCL-R Psychopathy Checklist-Revised, Shipley Wechsler Adult Intelligence Scale, WAS Welsh Anxiety Scale, $S R$ stress reaction 
Interview and self-report measures

Psychopathy Checklist-Revised To assess psychopathy, the Psychopathy Checklist-Revised (PCL-R; Hare, 2003) was used. Each of the 20 items can be rated 0 , 1, or 2, to indicate whether the trait is absent, somewhat present, or present, respectively. Information for the ratings was gathered through a semistructured interview and institutional file review. The inter-rater reliability (ICC) for PCL-R total scores, Factor 1, and Factor 2 was .97, .92, and .95, respectively, for the 13 inmates with dual PCL-R ratings.

Multidimensional Personality Questionnaire-Brief To assess anxiety, participants completed the Multidimensional Personality Questionnaire-Brief (MPQ-B; Patrick, Curtain, \& Tellegen, 2002), a 155 item self-report questionnaire that consists of 11 primary-trait scales. The Stress Reaction (SR) subscale is one of three main facets of the broad trait scale Negative Emotionality (NEM), with high scores related to tension, nervousness, and worry. Conversely, individuals with low SR do not feel vulnerable, can put fears and worries out their minds, get over upsetting experiences quickly, and are not troubled by emotional turmoil or guilty feelings (see Tellegen \& Waller, 1992). Thus, the SR scale captures both ends of the anxiety continuum. The internal consistency for the SR scale in this sample is .872 .

Welsh Anxiety Scale The Welsh Anxiety Scale (WAS; Welsh, 1956) is based on a factor analysis of the MMPI items and measures trait symptoms of anxiety, as well as general maladjustment (Graham, Barthlow, Stein, \& Ben-Porath, 2002). The internal consistency for the WAS in this sample is .926.

\section{Instructed fear-conditioning task}

The presentation of all stimuli and measurement of behavioral responses were controlled by DMDX software (Forster \& Forster, 2003). The instructed fear-conditioning task consisted of four conditions, with 80 trials per condition. We crossed focus of attention (threat focus or alternative focus) with the timing of this attentional focus (early onset or late onset) to yield the following conditions: early threat focus, late threat focus, early alternative focus, and late alternative focus. In the threat-focus conditions, participants had to focus on the color of a box that predicted shock administration. In the alternative-focus conditions, participants had to focus on whether a letter stimulus was capitalized (i.e., a threat-irrelevant aspect of the trial). Participants were told what aspect of the trial to focus on and respond to in instructions that appeared before each block. In addition, the timing of the focus of attention (early or late) was orthogonally manipulated to examine the effects of early onset versus late onset of the attentional focus. This was accomplished by presenting the taskrelevant stimulus either first or second in the trial sequence. For example, in the early-threat-focus condition, the colored box was presented first, followed by the letter stimulus. The order of conditions was counterbalanced across participants but did not interact significantly with the effects reported.

Every trial in all conditions started with a fixation cross lasting $200 \mathrm{~ms}$. Then, participants saw two stimuli presented sequentially. These stimuli consisted of a box (colored red or green) and a letter (an uppercase $\mathrm{N}$ or a lowercase $n$ ). Their order of appearance varied with the condition. The first stimulus appeared alone at $200 \mathrm{~ms}$; then, the second stimulus appeared concurrently with the first at $400 \mathrm{~ms}$. Following the offset of the stimuli, a blank screen appeared. At 1,800 ms, a descriptive word appeared on the screen, prompting participants to respond on the basis of either the first or the second stimulus, according to the condition. In the threat-focus conditions, the words "Red" or "Green" were presented, and participants pressed one of two buttons to indicate whether the word described the color of the box during that trial. In the alternative-focus conditions, the words "Upper" or "Lower" appeared, and participants pressed one of two buttons to indicate whether the word described the case of the letter during that trial. The response prompts lasted for $800 \mathrm{~ms}$ and were followed by an intertrial interval of $1,000 \mathrm{~ms}$. The time between the onset of the first stimulus on successive trials was 3,600 ms. Figure 2 summarizes trial timing and provides an example of a trial in each condition.

In all conditions, participants were told that electric shocks could be administered following presentation of a red box (threat trial), but that shocks would never occur following presentation of a green box (no-threat trial). An electric shock was administered for $200 \mathrm{~ms}$ to two adjacent fingers on each participant's nondominant hand on $15 \%$ of threat trials in each condition. These shocks were delivered at $1,600 \mathrm{~ms}$ into the trial. A total of 24 shocks (six per condition) were administered to each participant, and the intensity of shocks was calibrated to participants' subjective tolerance. The case of the letter stimulus ( $\mathrm{N}$ or $\mathrm{n}$ ) was unrelated to the administration of electric shocks. Finally, participants were told they could earn up to $\$ 3$ in additional pay according to the speed and accuracy of their responses across all four blocks.

\section{Shock sensitivity evaluation}

To control for individual differences in shock sensitivity, the intensity of shocks received during the instructed fear task was calibrated to the participants' individual subjective shock sensitivity prior to the beginning of the task. A series 
A. Early Alternative Focus

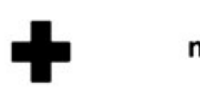

n

B. Late Alternative Focus
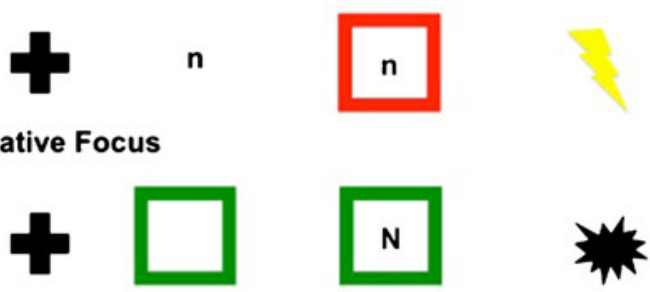

Upper

Mismatch

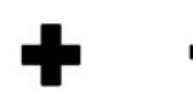

n

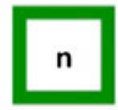

D. EarlyThreat Focus
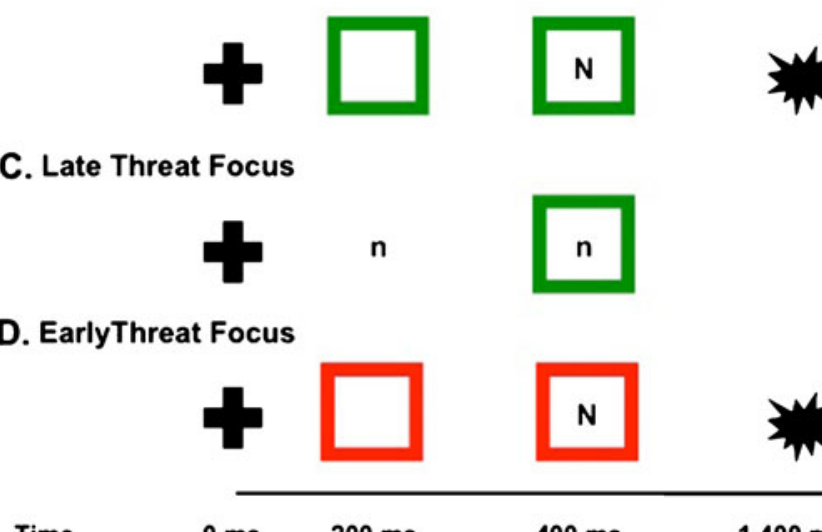

Upper

Match

\section{Late Threat Focus}

Red

\section{Time
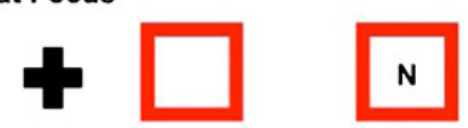 \\ $0 \mathrm{~ms}$ \\ $200 \mathrm{~ms}$ \\ $400 \mathrm{~ms}$ \\ Fixation \\ Stimulus 1 \\ Stimulus 2}

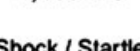

Blank Screen

Mismatch

Fig. 2 Schematic of task. Trial structure in the four conditions used in the experiment. Every trial began with a fixation cross lasting $200 \mathrm{~ms}$, after which participants saw two stimuli: a box (colored red or green) and a letter (an uppercase $N$ or a lowercase $n$ ). The order of these two stimuli varied with condition. The first stimulus appeared alone at $200 \mathrm{~ms}$, and then the second stimulus appeared concurrently with the first at $400 \mathrm{~ms}$. In all four conditions, electric shocks were administered after some red boxes but never after green boxes.

of electric shocks was administered in increasing intensity to the fingers of their nondominant hand. Participants reported two intensity anchors: the first, intensity that they considered uncomfortable, and then the maximum intensity level that they could tolerate. The series was terminated when they reached their maximum intensity level. The shock intensity administered during the experimental session was calibrated to the reported maximum intensity level.

Startle response elicitation and measurement

Sixty-four startle-eliciting noise probes $(50 \mathrm{~ms}, 102 \mathrm{~dB}$ white noise burst with near instantaneous rise time) were presented 1,600 ms after the onset of the first stimulus. The noise probes were equally distributed across threat/no-threat trials in all four task conditions so that each participant received 16 noise probes (eight threat and eight no-threat) per task condition. Noise probes were also equally distributed across upper/lower case trials. Noise probes were separated by a minimum of $13 \mathrm{~s}$ and never occurred on the same trial as shock administration. Startle eyeblink electromyographic activity was sampled and amplified with
Following the offset of the stimuli, a blank screen appeared. White-noise startle probes were presented at 1,600 ms into the trial (i.e., during the blank screen) to measure fear-potentiated startle. At $1,800 \mathrm{~ms}$, a descriptive word related to the color of the box or the case of the letter (i.e., "Green" or "Red" for threat-focus blocks; "Upper" or "Lower" for alternative-focus blocks) appeared on the screen. Participants had to indicate whether the word matched (or mismatched) the relevant feature presented during that trial

Neuroscan Synamps2 amplifiers (Comupmedics Inc., North Carolina) at $2,000 \mathrm{~Hz}$ with a bandpass filter $(30-500 \mathrm{~Hz}$; $24 \mathrm{~dB} /$ octave roll-off) from electrodes placed on the skin overlaying the orbicularis oculi muscle under the right eye according to published guidelines (Blumenthal, Cuthbert, Filion, Hackley, Lipp, \& van Boxtel, 2005). Offline processing included epoching ( $-50 \mathrm{~ms}$ to $250 \mathrm{~ms}$ surrounding noise probe), rectification and smoothing (30-Hz lowpass filter following rectification), and baseline correction. Startle blink magnitude was scored as the peak response between a $20-120$ ms post-probe onset. Fear response to threat cues was indexed by FPS (measured in microvolts), calculated as the difference in blink-response magnitude to probes following red (threat) versus green (no-threat) boxes in each of the four conditions.

Data analysis

FPS was analyzed using a general linear model (GLM) with total psychopathy, SR scores, and their interaction as between-subjects factors and the four conditions as a within-subjects variable. In order to specify which conditions were uniquely related to the fear response, we used 
three orthogonal Helmert contrasts that compared (a) the early alternative focus condition with the average of the other three conditions, (b) the late alternative focus condition with the average of the two threat focus conditions, and (c) the late threat focus with the early threat focus condition. Our use of the four-condition within-subjects variable parallels the strategy employed by Baskin-Sommers et al. (2011), as does our use of three orthogonal Helmert contrasts to specify the significant condition effects.

\section{Results}

None of the main effects included in the GLM approached statistical significance. These findings indicate that FPS was comparable across all four task conditions $(p=.876)$, and that neither psychopathy $(p=.209)$ nor SR scores ( $p=.699)$ was consistently related to FPS across task conditions. The psychopathy by SR interaction was also nonsignificant $(p=.509)$. However, the psychopathy $\mathrm{x}$ condition interaction, $F(3,249)=3.38, p=.019, \mathrm{p \eta}^{2}=.039$, and the anxiety x condition interaction, $F(3,249)=2.66$, $p=.049, \mathrm{p}^{2}=.031$, were both statistically significant.

Clarifying the significant psychopathy $\mathrm{x}$ condition interaction, the three independent Helmert contrasts indicated that this interaction was due to the psychopathyrelated differences in the early alternative focus conditions being significantly greater than the psychopathy-related differences in the other three conditions, $F(1,83)=7.86$, $p=.006, \mathrm{p \eta}^{2}=.086$. These analyses and results are essentially the same as those described by Baskin-Sommers et al. (2011). The fact that the results for psychopathy were essentially unchanged by inclusion of anxiety in the GLM model indicates that psychopathy and anxiety do not explain common variance in FPS and that anxiety did not mediate the significant effect of psychopathy on FPS. See Table 2 for the GLM point estimates for raw startle and FPS by Psychopathy, SR and Condition. ${ }^{3}$

To clarify the significant anxiety $\mathrm{x}$ condition interaction, we conducted the same three orthogonal (Helmert) contrasts to unpack the significant psychopathy $\mathrm{x}$ condition interaction. The first contrast compared the relationship between anxiety and FPS in the early alternative-focus condition versus the other three conditions. (Note: This contrast was significant for psychopathy [Baskin-Sommers

\footnotetext{
${ }^{3}$ To provide additional information, we repeated this analysis using PCL-R Factor 1 and then PCL-R Factor 2 in place of PCL-R total scores in the overall analyses. In both cases, the individual PCL-R factors replicated the basic findings. In other words, each dimension was associated with significantly less FPS in the early alternative focus condition relative to the others and the effects for SR reported in the manuscript were essentially unchanged.
}

et al., 2011]). The second interaction contrast compared the late alternative-focus condition with the two threat-focus conditions, and the third interaction contrast compared the two threat-focus conditions. Neither of the first two interaction contrasts was significant $(p=.66$ and $p=.56$ ). However, the third contrast involving the late threatfocus and early threat-focus conditions was significant, $F(1,83)=6.93, p=.01, \mathrm{p \eta}^{2}=.077$ (see Fig. 3). Followup simple-effects tests revealed that anxiety was positively related to FPS in the early threat-focus condition (B = $5.50, p=.049)$ and was negatively, albeit nonsignificantly, related to FPS in the late threat-focus condition ( $\mathrm{B}=$ $-3.02, p=.38$ ).

Finally, we examined the three-way interaction involving psychopathy, anxiety, and condition to determine whether anxiety moderated the association between psychopathy and FPS. This interaction did not approach statistical significance, $F(3,249)=1.24, p=.30$. Thus, anxiety did not moderate the effects of psychopathy on FPS in this study.

\section{Supplementary analyses}

In the RST literature, trait anxiety has been conceptualized as a combination of trait neuroticism and introversion, with the weighting of neuroticism (.66) being approximately twice as large as that for introversion (.33; see Corr, 2008; Gray, 1987). This characterization of anxiety corresponds closely to traditional self-report measures of neurotic anxiety, such as the SR and WAS used in this study. In addition, such measures are generally highly correlated and relatively interchangeable (see Watson \& Clark, 1984). The correlation between SR and WAS in the current sample was $r(87)=.739$. To examine the generalizability of our findings, we repeated our analyses using the WAS in place of the SR scale. Although the overall findings exhibited the same pattern, they were generally weaker for WAS than for the SR scale. Of particular relevance for evaluating the generalizability of our finding, the anxiety $\mathrm{x}$ condition contrast comparing the two threat-focus conditions was no longer statistically significant, $p=.158$.

Despite the high correlation between our two measures of trait anxiety, the discrepant findings for the two measures suggest a potential problem with the reliability of our anxiety assessments. A common method for improving the reliability of related assessments is to combine the measures to yield a single more reliable assessment. Thus, we $z$ scored and combined the SR and WAS measures of anxiety to produce a more reliable composite measure of anxiety. When the data were reanalyzed using this composite measure of trait anxiety, the results replicated the overall pattern reported for the SR scale. Specifically, the interaction contrast comparing the two threat-focus conditions was significant, $p=.04$, whereas the other two interaction 
Table 2 General linear model point estimates (and standard errors) for startle response associated with no-threat and threat cues and fearpotentiated startle (FPS) as a function of stress reduction (SR) and condition

\begin{tabular}{|c|c|c|c|c|c|c|c|c|c|c|c|}
\hline \multicolumn{3}{|c|}{ Early alternative-focus } & \multicolumn{3}{|c|}{ Late alternative-focus } & \multicolumn{3}{|c|}{ Late threat-focus } & \multicolumn{3}{|c|}{ Early threat-focus } \\
\hline No threat & Threat & FPS & No threat & Threat & FPS & No threat & Threat & FPS & No threat & Threat & FPS \\
\hline \multicolumn{12}{|l|}{ Low SR } \\
\hline $45.43(8.14)$ & $59.57(12.01)$ & $14.14(6.97)$ & $41.45(8.59)$ & $59.29(11.07)$ & $17.84(5.21)$ & $38.09(8.14)$ & $61.77(10.76)$ & $23.69(6.02)$ & $42.76(8.60)$ & $52.06(11.13)$ & $9.29(4.98)$ \\
\hline \multicolumn{12}{|l|}{ High SR } \\
\hline $50.71(8.14)$ & $70.16(12.01)$ & $19.44(6.97)$ & $46.94(8.59)$ & $63.35(11.07)$ & $16.41(5.21)$ & $48.53(8.14)$ & $63.03(10.76)$ & $14.51(6.02)$ & $47.81(8.60)$ & $72.73(11.13)$ & $24.92(4.98)$ \\
\hline \multicolumn{12}{|l|}{ Condition means } \\
\hline $48.07(4.48)$ & $64.87(6.61)$ & $16.80(3.78)$ & $44.19(4.72)$ & $61.32(6.08)$ & $17.13(2.86)$ & $43.31(4.84)$ & $62.41(5.19)$ & $19.10(3.32)$ & $45.29(4.73)$ & $62.39(6.16)$ & $17.10(2.79)$ \\
\hline
\end{tabular}

Fear response to threat cues was indexed by FPS, which is calculated by subtracting blink-response magnitude to probes following green (nothreat) boxes from probes following red (threat) boxes in each of the four conditions. Because we analyzed SR continuously instead of using an extreme groups design, the values presented are point estimates (i.e., estimated using regression analyses) for low SR (1.5 standard deviations below sample mean) and high SR (1.5 standard deviations above sample mean) points on the distribution

contrasts remained nonsignficant (contrast $1, p=.74$; and contrast $2, p=.59$ ). Also paralleling the results of our initial analysis, the first interaction contrast involving psychopathy remained significant and essentially unchanged ( $p=.005$ ), confirming that the effect of psychopathy is essentially independent of trait anxiety.

\section{Discussion}

In contrast with psychopathic individuals who are notoriously insensitive to threat cues and are prone to disinhibited

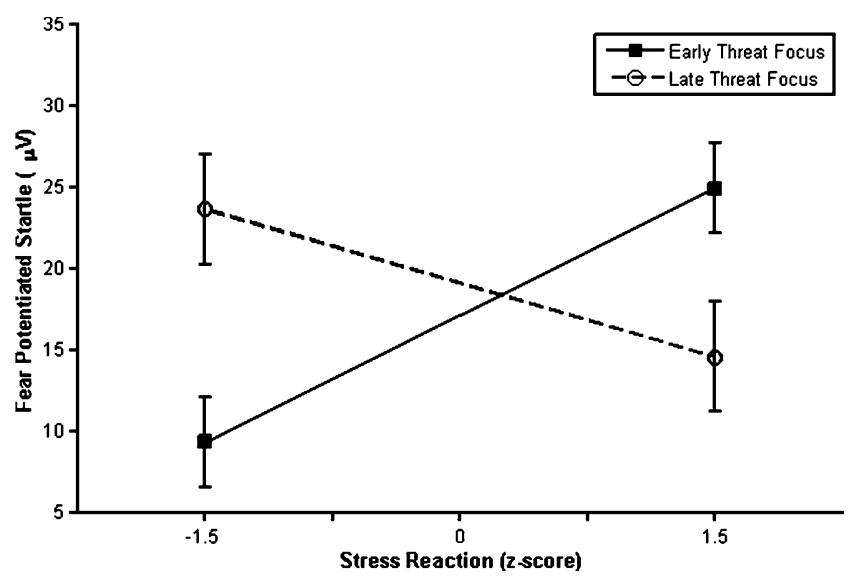

Fig. 3 Fear-potentiated startle (FPS) by anxiety and condition. FPS was analyzed in a general linear model (GLM) with condition as a within-subjects categorical factor and SR and psychopathy total score (mean-centered and standardized) as between-subjects continuous factors. The relationship between SR and FPS was moderated by condition. SR was positively related to FPS in the early threat-focus condition ( $\mathrm{B}=5.50, p=.049)$, and was negatively, albeit nonsignificantly, related to FPS in the late threat-focus condition $(\mathrm{B}=-3.02, p=.38$ ). FPS (in microvolts $[\mu \mathrm{V}]$ ) was calculated as startle response during red minus green box trials. FPS is displayed for the range of SR scores from \pm 1.5 standard deviations from the mean. Error bars represent \pm one standard error for FPS point estimates for low and high SR behavior, anxious individuals are hypersensitive to threat cues and are overly inhibited. To examine whether these characteristics involve a common mechanism operating in opposite directions or independent mechanisms, we examined the effects of trait anxiety on sensitivity to threat cues using the same participants and paradigm used to assess threat sensitivity in psychopathic individuals. As was expected, trait anxiety was associated with significantly greater FPS (i.e., sensitivity to threat cues). However, this effect was condition specific and occurred in a different condition than the one that revealed psychopathy-related deficits in FPS. Moreover, our analyses revealed that the psychopathy effects reported by Baskin-Sommers et al. (2011) were virtually unchanged by the simultaneous inclusion of anxiety, demonstrating the statistical independence of the psychopathy and anxiety effects. Thus, at least with regard to the threat sensitivity evaluated in this paradigm, the fearlessness of psychopathic offenders is not mediated by anxiety and, in fact, the effects of psychopathy and anxiety on sensitivity to threat cues appear to be statistically independent.

The fact that significant psychopathy-related and anxiety-related differences in FPS were found under different conditions provides an unusual opportunity to compare and contrast the factors underlying their differential sensitivity to threat stimuli. Of particular interest, the manipulation of threat onset and threat relevancy makes it possible to evaluate the extent to which psychopathy and anxiety reflect different processes. For example, the alternative-focus conditions provide a measure of threat sensitivity when threat is peripheral to participants' goaldirected focus of attention and, thus, correspond to the revised model of BIS functioning described by Gray and McNaughton (2000) (i.e., readiness to interrupt approach behavior to process peripheral threat cues). On the other hand, the threat-focus conditions assess sensitivity to threat directly and, thus, correspond to the FFFS in the revised 
RST framework (i.e. threat sensitivity). It is important to note that our use of the RST framework is intended to provide a theoretical context for distinguishing separable threat-processing mechanisms rather than reify the hypothetical BIS and FFFS constructs and their associations with psychopathy and anxiety. Although these constructs and associations will evolve over time (see Footnote 1), such changes would not alter the primary implications of our findings: that psychopathy- and anxiety-related differences in threat sensitivity were found to be statistically independent. Consistent with the response modulation model, psychopathy-related deficits in FPS were specific to the early-alternative focus condition that presented goalrelevant information before threat-relevant information, as detailed by Baskin-Sommers et al. (2011). Conversely, trait anxiety was associated with significantly stronger FPS in the early threat-focus condition, indicating that trait anxiety is associated with a greater sensitivity to threat stimuli, particularly when threat is the primary focus of attention and the first stimulus presented.

In contrast with the early threat-focus condition, anxious individuals showed relatively weak FPS in the late threatfocus condition. Although this pattern was unexpected, Lang, Bradley, and Cuthbert's (1997) defense cascade model suggests a potential explanation for this effect (see also Bradley, Codispoti, Cuthbert, \& Lang, 2001). Specifically, this model suggests two stages of defensive action. The first stage is associated with an inhibition of motor responses during sensory intake and attentional allocation, resulting in a decrease in the startle response. In the second stage, as more elaborative threat processing occurs, arousal increases, and startle is potentiated as fear structures (i.e., the amygdala) prepare for defensive action. Related to this logic, startle probes presented shortly after aversive pictures are associated with weaker startle, as compared with a longer probe delay (Bradley, Cuthbert, \& Lang, 1993). In the late threat-focus condition, irrelevant letters were presented prior to the threat-relevant colored boxes. To the extent that anxious participants have a perceptual bias toward threat ( $\mathrm{Li}$ et al., 2008), the preceding irrelevant distractor may have interrupted their threat orienting, resulting instead in orientation toward the irrelevant distractor (i.e., to determine whether this was threat information), and delaying the detection and processing of threat. Thus, the functioning of defense cascade was initiated only when the colored box appeared. However, because this cue was temporally closer to the startle probe, it is likely that participants were still engaged in the attention orienting stage, in which startle is suppressed.

The fact that anxiety was not associated with FPS in the alternative focus conditions appears to be at odds with Dvorak-Bertsch et al.'s (2007) results indicating greater FPS among high anxious undergraduates in their alternative focus condition. Similarly, Bishop, Jenkins, and Lawrence (2007) also found that high anxiety was associated with greater amygdala activation to peripheral threat distracters, though particularly under conditions of low perceptual load. Moreover, Bishop and colleagues attribute the heightened threat reactivity of anxious individuals to reduced "recruitment of [prefrontal] control mechanisms used to prevent the further processing of salient distractors" (p. 1595; see also Bishop, 2009). Overall, these findings suggest that anxious individuals have difficulty employing cognitive control and disengaging attention from threat information (Fox, Russo, Bowles, \& Dutton, 2001; Fox, Russo \& Dutton, 2002). Such evidence might suggest that in the present study, and consistent with Dvorak-Bertsch et al. (2007), anxious individuals should have displayed greater FPS under the alternative-focus conditions.

Importantly, though, there are numerous methodological differences that distinguish the present study from past research. A potentially crucial difference concerns the sequential presentation of goal-relevant and distracter stimuli. In both the Bishop (2009; Bishop et al., 2007) and Dvorak-Bertsch et al. (2007) studies, the goal-relevant and threat-relevant stimuli were presented simultaneously, increasing the likelihood that both elements of the display would be processed initially, and thus increasing the importance of prefrontal attention control. Conversely, in the present study, the need for prefrontal control may have been minimized by the discrete trial procedure, the perceptual discriminability of distracter versus goalrelevant stimuli (i.e., colored box vs. letter), and the temporal separation of goal-relevant and distracter stimuli. One possibility is that these and other methodological differences (e.g., timing of FPS assessment) created an experimental context that was suboptimal for measuring the anxiety-related deficit in attention control described by Bishop and colleagues.

Despite this, the present findings are consistent with the theoretical approach that suggests that trait-anxious individuals are characterized by exaggerated threat sensitivity. In general, high-anxious individuals may orient toward or respond more strongly to threat-related stimuli, resulting in greater amygdala activation (Bishop, 2009) and/or greater activation of the FFFS (Corr, 2008). Specific to this study, results suggest that under conditions in which the threat is prepotent, this strong threat response is exacerbated. In addition, the allocation of attention to salient emotion cues may impair other executive functions, including inhibition, shifting, updating, and control (Deveney \& Pizzagalli, 2008; Ellis \& Ashbrook, 1988; Keil, Bradley, Junghöfer, Russmann, Lowenthal \& Lang, 2007; Pessoa, 2009). That is, a pervasive threat bias of this type could undermine a person's capacity to maintain cognitive control when one's bias to process emotion distractors competes with an 
experimenter's instructions to focus on a competing set of stimuli (e.g., Bishop, Duncan, Brett, \& Lawrence, 2004; Dvorak-Bertsch et al., 2007). Similarly, such a bias may also work against reorienting attention away from salient threat stimuli, as was reported by Fox and colleagues (Fox et al., 2001, 2002).

As was already described, a primary purpose of the present study was to compare and, if possible, to extract the unique contributions of psychopathy and anxiety to threat sensitivity. Moreover, in the event that psychopathy and anxiety were found to reflect independent rather than overlapping influences on threat sensitivity, we reasoned that the two dimensions might interact to determine threat sensitivity. As was illustrated by the present results, psychopathy and anxiety do not always interact to determine behavior. Nevertheless, we propose that the independent mechanisms identified in the present study may provide a meaningful framework for understanding previously reported psychopathy by anxiety interactions. To illustrate the point, we briefly consider two examples.

Numerous studies have demonstrated that passive avoidance learning deficits are more pronounced in low-anxious psychopathic offenders than in high-anxious psychopathic offenders (e.g., Newman, Patterson, Howland, \& Nichols, 1990; Newman \& Schmitt, 1998). Given the present findings, one interpretation of such findings is that the heightened threat sensitivity associated with high anxiety may compensate for the weak response modulation of psychopathic individuals. In other words, anxiety appears to moderate (i.e., interacts with) the otherwise general tendency of psychopathic offenders to over-respond for reward despite being punished for inappropriate responses. Other studies have found that the strong behavioral inhibition and electrodermal reactivity to threat stimuli that generally characterizes high anxious individuals is substantially diminished in participants who also have high psychopathy scores (e.g., Arnett et al., 1997; Newman et al., 1997). Alternatively, in light of the present findings, it may be that the response modulation deficits associated with psychopathy may protect anxious individuals from overreacting to threat-related information. Our interpretation of these findings is speculative and provided primarily for purposes of illustration. Clearly, further research is needed to clarify the circumstances that are differentially associated with psychopathy-related versus anxiety-related differences in threat sensitivity and to specify the circumstances that give rise to psychopathy by anxiety interactions.

Before concluding, it is important to consider potential limitations of this study. First, the fact that the study was conducted in a male prison raises legitimate concerns about the applicability of our findings to the general population. Thus, it is important to replicate these findings in a nonincarcerated, mixed-gender sample. Nevertheless, because the PCL-R was designed to assess psychopathy within prisons and a secondary goal of this investigation was to address the interaction between psychopathy and anxiety within prison samples, we believe that the costs associated with using a prison sample are outweighed by the benefits of using this well-validated measure of psychopathy. Second, although similar, our analyses examining the generalizability of our findings to a second measure of trait anxiety (i.e., the WAS) fell short of statistical significance. Nonetheless, the fact that we were able to replicate the SR findings using a composite measure of anxiety suggests that the use of a broader and/or more reliable measure of trait anxiety would yield more consistent findings. Finally, it is possible that the statistically independent and distinct effects of anxiety and psychopathy found in this study are specific to the paradigm that we employed. Although there are many advantages to our paradigm for parsing the mechanisms associated with psychopathy and anxiety, research with other paradigms is needed to determine the extent to which anxiety and psychopathy are reliably associated with heightened sensitivity to threat cues (i.e., FFFS functioning) and reduced inclination to suspend goal-directed behavior to process threat-related stimuli (i.e., poor response modulation, weak BIS functioning), respectively.

In sum, consistent with previous clinical and empirical characterizations of the constructs, we found that psychopathy and anxiety are associated with low and high threat sensitivity, respectively. However, our results suggest that it may be inaccurate to assume that this difference reflects opposite ends on a single dimension or a common underlying process. To the contrary, we found that psychopathy-related and anxiety-related differences in threat sensitivity occurred under different experimental conditions and were statistically independent, suggesting that they involve distinct psychobiological mechanisms. Additional research is needed to specify the attention-emotion interactions that account for psychopathy-related versus anxiety-related differences in threat sensitivity and to investigate the generalizability of our findings to other samples and experimental paradigms.

\section{References}

Arnett, P. A., Smith, S. S., \& Newman, J. P. (1997). Approach and avoidance motivation in incarcerated psychopaths during passive avoidance. Journal of Personality and Social Psychology, 72, $1413-1428$.

Baskin-Sommers, A. R., Curtin, J. J., \& Newman, J. P. (2011). Specifying the attentional selection that moderates the fearless- 
ness of psychopathic offenders. Psychological Science, 22(2) 226-234.

Baskin-Sommers, A., Wallace, J., MacCoon, D., Curtin, J., \& Newman, J. (2010). Clarifying the factors that undermine behavioral inhibition system functioning in psychopathy. Personality Disorders: Theory, Research, and Treatment, 1, 203-217.

Bishop, S. J. (2009). Trait anxiety and impoverished prefrontal control of attention. Nature Neuroscience, 12, 92-98.

Bishop, S. J., Duncan, J., Brett, M., \& Lawrence, A. (2004). Prefrontal cortical function and anxiety: Controlling attention to threatrelated stimuli. Nature Neuroscience, 7, 184-8.

Bishop, S. J., Jenkins, R., \& Lawrence, A. (2007). Neural processing of fearful faces: Effects of anxiety are gated by perceptual capacity limitations. Cerebral Cortex, 17, 1595-1603.

Blumenthal, T. D., Cuthbert, B. N., Filion, D. L., Hackley, S., Lipp, O. V., \& van Boxtel, A. (2005). Committee report: Guidelines for human startle eyeblink electromyographic studies. Psychophysiology, $42,1-15$.

Bradley, M. M., Codispoti, M., Cuthbert, B. N., \& Lang, P. J. (2001). Emotion and motivation I: Defensive and appetitive reactions in picture processing. Emotion, 1, 276-298.

Bradley, M. M., Cuthbert, B. N., \& Lang, P. J. (1993). Pictures as prepulse: Attention and emotion in startle modification. Psychophysiology, 30, 541-545.

Cleckley, H. M. (1976). The mask of sanity (5th ed.). St. Louis, MO: Mosby.

Corr, P. J. (Ed.). (2008). The reinforcement sensitivity theory of personality. New York, NY: Cambridge University Press.

Deveney, C. M., \& Pizzagalli, D. A. (2008). The cognitive consequences of emotion regulation: An ERP investigation. Psychophysiology, 45, 435-444.

Dvorak-Bertsch, J. D., Curtin, J. J., Rubinstein, T. J., \& Newman, J. P. (2007). Anxiety moderates the interplay between cognitive and affective processing. Psychological Science, 18, 699-705.

Ellis, H. C., \& Ashbrook, P. W. (1988). Resource allocation model of the effects of depressed mood states on memory. In K. Fiedler \& J. Forgas (Eds.), Affect, cognition, and social behaviour (pp. 25-43). Toronto, Canada: Hogrefe.

Forster, K. I., \& Forster, J. C. (2003). DMDX: A Windows display program with millisecond accuracy. Behavioral Research Methods, 35, 116-124.

Fowles, D. C. (1980). The three arousal model: Implications of Gray's two-factor learning theory for heart rate, electrodermal activity, and psychopathy. Psychophysiology, 17, 87-104.

Fowles, D. C. (2001). Biological variables in psychopathology: A psychobiological perspective. In P. Sutker \& H. Adams (Eds.), Comprehensive handbook of psychopathology (pp. 85-104). New York, NY: Kluwer Academic/Plenum.

Fox, E., Russo, R., Bowles, R., \& Dutton, K. (2001). Do threatening stimuli draw or hold attention in subclinical anxiety? Journal of Experimental Psychology. General, 130, 681-700.

Fox, E., Russo, R., \& Dutton, K. (2002). Attentional bias for threat: Evidence for delayed disengagement from emotional faces. Cognition \& Emotion, 16, 355-379.

Graham, J. R., Barthlow, D. L., Stein, L. A. R., \& Ben-Porath, Y. S. (2002). Assesing general maladjustment with the MMPI-2. Journal of Personality Assessment, 78, 334-347.

Gray, J. A. (1972). Learning theory, the conceptual nervous system, and personality. In V. D. Nebylitsyn \& J. A. Gray (Eds.), Biological basis of individual behavior (pp. 182-205). New York, NY: Academic Press.

Gray, J. A. (1975). Elements of a two-process theory of learning. New York, NY: Academic Press.

Gray, J. A. (1982). The neuropsychology of anxiety: An inquiry into the functions of the septo-hippocampal system (1st ed.). New York, NY: Oxford University Press.
Gray, J. A. (1987). The psychology of fear and stress. New York, NY: Cambridge University Press.

Gray, J. A., \& McNaughton, N. (2000). The neuropsychology of anxiety: An inquiry into the functions of the septo-hippocampal system (2nd ed.). New York, NY: Oxford University Press.

Hare, R. D. (1965). Temporal gradient for fear arousal in psychopaths. Journal of Abnormal Psychology, 70, 442-445.

Hare, R. D. (2003). Manual for the hare psychopathy checklistRevised (2nd ed.). Toronto, Canada: Multi-Health Systems.

Hare, R. D., Harpur, T. J., \& Hakstian, A. R. (1990). The revised psychopathy checklist: Reliability and factor structure. Psychological Assessment, 2, 338-341.

Hart, S. D., \& Hare, R. D. (1989). Discriminant validity of the Psychopathy Checklist in a forensic psychiatric population. Psychological Assessment: A Journal of Consulting and Clinical Psychology, 1, 211-218.

Keil, A., Bradley, M. M., Junghöfer, M., Russmann, T., Lowenthal, W., \& Lang, P. J. (2007). Cross-modal attentional capture by affective stimuli. Cognitive, Affective \& Behavioral Neuroscience, 7, 18-24.

Lang, P. J., Bradley, M. M., \& Cuthbert, B. N. (1997). Motivated attention: Affect, activation, and action. In P. Lang, R. Simons, \& M. Balaban (Eds.), Attention and orienting: Sensory and motivational processes (pp. 97-135). Mahwah, NJ: Erlbaum.

Li, W., Zinbarg, R. E., Boehm, S. G., \& Paller, K. A. (2008). Neural and behavioral evidence for affective priming from unconsciously perceived emotional facial expressions and the influence of trait anxiety. Journal of Cognitive Neuroscience, 20, 95-107.

MacLeod, C., Mathews, A., \& Tata, P. (1986). Attentional bias in emotional disorders. Journal of Abnormal Psychology, 95, 15-20.

Mathews, A., \& MacLeod, C. (2002). Induced emotional biases have causal effects on anxiety. Cognition and Emotion, 16, 310-315.

Mathews, A., \& MacLeod, C. (2005). Cognitive vulnerability to emotional disorders. Annual Review of Clinical Psychology, 1, 167-95.

Mogg, K., \& Bradley, B. P. (1998). A cognitive-motivational analysis of anxiety. Behaviour Research and Therapy, 36, 809-848.

Newman, J. P., Curtin, J. J., Bertsch, J. D., \& Baskin-Sommers, A. R. (2010). Attention moderates the fearlessness of psychopathic offenders. Biological Psychiatry, 67, 66-70.

Newman, J. P., \& Lorenz, A. R. (2003). Response modulation and emotion processing: Implications for psychopathy and other dysregulatory psychopathology. In R. J. Davidson, K. Scherer, \& H. H. Goldsmith (Eds.), Handbook of affective sciences (pp. 904-929). New York, NY: Oxford University Press.

Newman, J. P., Patterson, C. M., Howland, E. W., \& Nichols, S. L. (1990). Passive avoidance in psychopaths: The effects of reward. Personality and Individual Differences, 11, 1101-1114.

Newman, J. P., \& Schmitt, W. A. (1998). Passive avoidance in psychopathic offenders: A replication and extension. Journal of Abnormal Psychology, 107, 527-532.

Newman, J. P., Wallace, J. F., Schmitt, W. A., \& Arnett, P. A. (1997). Behavioral inhibition system functioning in anxious, impulsive, and psychopathic individuals. Personality and Individual Differences, 23, 583-592.

Patrick, C. J., Curtin, J. J., \& Tellegen, A. (2002). Development and validation of a brief form of the Multidimensional Personality Questionnaire. Psychological Assessment, 14, 150-163.

Patterson, C. M., \& Newman, J. P. (1993). Reflectivity and learning from aversive events: Toward a psychological mechanism for the syndromes of disinhibition. Psychological Review, 100, 716-736.

Pessoa, L. (2009). How do emotion and motivation direct executive control? Trends in Cognitive Science, 13, 160-166.

Ross, S. R., Molto, J., Poy, R., Segarra, P., Pastor, M. C., \& Montanes, S. (2007). Gray's model and psychopathy: BIS but not BAS differentiates primary from secondary psychopathy in noninsti- 
tutionalized young adults. Personality and Individual Differences, 43, 716-736.

Schmitt, W. A., \& Newman, J. P. (1999). Are all psychopathic individuals low-anxious? Journal of Abnormal Psychology, 108, 353-358.

Tellegen, A., \& Waller, N. G. (1992) Exploring personality through test construction: Development of the Multi-dimensional Personality Questionnaire (MPQ). Unpublished manuscript. Department of Psychology, University of Minnesota.

Wallace, J. F., \& Newman, J. P. (2008). Reinforcement sensitivity theory and psychopathy: Associations between psychopathy and the behavioral activation and inhibition systems. In P. J. Corr (Ed.), Reinforcement Sensitivity Theory of Personality (pp 398414). Cambridge University Press.

Watson, D., \& Clark, L. A. (1984). Negative affectivity: The disposition to experience aversive emotional states. Psychological Bulletin, 96, 465-490.
Welsh, G. (1956). Factor dimensions A and R. In G. S. Welsh \& W. G. Dahlstrom (Eds.), Basic readings on the MMPI in psychology and medicine (pp. 264-281). Minneapolis: University of Minnesota Press.

Williams, J. M. G., Mathews, A., \& MacLeod, C. (1996). The emotional Stroop task and psychopathology. Psychological Bulletin, 120, 3-24.

Zachary, R. A. (1986). Shipley institute of living scale: Revised manual. Los Angeles, CA: Western Psychological Service.

\section{Author Note}

This work was supported by NIMH Grants 5R01MH053041 and T32MH018931. We thank many at the Wisconsin Department of Corrections for making this research possible. 